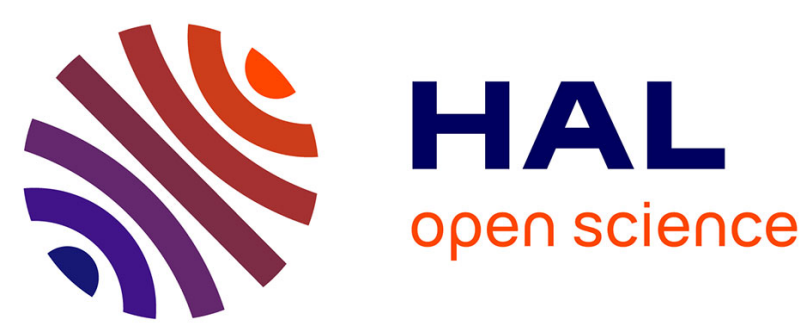

\title{
L'équilibre sylvocynégétique
}

Henri Daburon

\section{- To cite this version:}

Henri Daburon. L'équilibre sylvocynégétique. Revue forestière française, 1968, 9, pp.567-570. $10.4267 / 2042 / 20219$. hal-03385904

\section{HAL Id: hal-03385904 https://hal.science/hal-03385904}

Submitted on 19 Oct 2021

HAL is a multi-disciplinary open access archive for the deposit and dissemination of scientific research documents, whether they are published or not. The documents may come from teaching and research institutions in France or abroad, or from public or private research centers.
L'archive ouverte pluridisciplinaire HAL, est destinée au dépôt et à la diffusion de documents scientifiques de niveau recherche, publiés ou non, émanant des établissements d'enseignement et de recherche français ou étrangers, des laboratoires publics ou privés. 

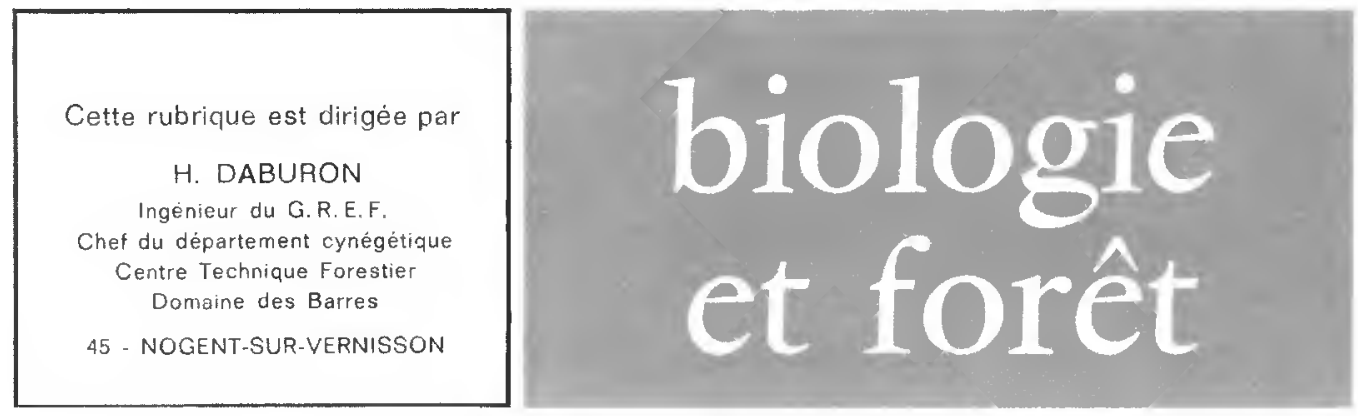

\title{
L'É Q U I I B R E S Y L O C Y N É G É T I Q U E
}

\author{
H. DABURON
}

Le jargon technico-administratif des forestiers s'est accru depuis quelques années de l'expression nouvelle d' "équilibre sylvocynégétique ".

Comme beaucoup de ses semblables, ce terme à la consonance quelque peu pédante éveille la méfiance des non-initiés : ils craignent que l'on n'ait voulu voiler le problème immédiatement perceptible des dégâts de gibier sous quelque fumeuse conception sans débouché pratique. A vrai dire, les anathèmes que se jettent réciproquement depuis des lustres forestiers et chasseurs rendent impensable. sinon cocasse pour certains. l'idée même de juxtaposer en un seul adjectif deux activités aussi divergentes à leurs yeux que la chasse et la foresterie et, a fortiori. de parler d'équilibre.

Cependant, on ne voit pas pourquoi cette question ne pourrait pas être abordée sans passion excessive, et pourquoi on ne pourrait y appliquer un raisonnement méthodique. Nous nous y emploierons dans la suite de cet article et, en premier líeu, c'est la conception même d'équllibre sylvocynégétique que nous essaierons de cerner.

\section{La conception d'équilibre sylvocynégétique}

Remarquons pour commencer que, tel que nous l'entendons, l'équilibre sylvocynégétique n'a que fort peu de rapports avec l'équilibre qui s'établissait naturellement de lui-même, avant l'invention de l'agriculture, du temps oú nos athlétiques ancêtres chassajent l'auroch à l'épieu ou au coup de poing de silex.

A cette époque l'Homme, d'ailleurs peu abondant, ètait un prédateur parmi d'autres; il servait parfois lui-même de proie et son impact sur la forêt, réduit à quelques cueillettes, était pratiquement nul, notamment sur les végétaux ligneux.

Un équilibre naturel s'établissait à l'intérieur du groupe Homme-Carnivores-Herbivores et, par l'intermédiaire des Herbivores "consommateurs primaires ", entre ce groupe ef la sylve primitive.

Aujourd'hui, par contre, dans nos régions, l'Homme s'est érigé en arbitre de l'équilibre entre une forêt façonnèe selon ses propres besoins et un groupe d'animaux sauvages dont l'équilibre interne est lui-même détruit : il en modèle, en effet, à sa guise les différentes populations par l'élimination des grands Carnivores, la chasse des grands Herbivores suivant leur sexe ou leur conformation, la destruction des "nuisibles " et des petits carnassiers pour favoriser les populations de petit gibier, d'ailleurs souvent introduit (faisan). Si donc on peut considérer qu'il s'agit bien toujours d'un équilibre biologique, puisque l'Homme appartient à la communauté vivante, celui-ci s'y insère avec tout son cortège d'exigences financières, sociologiques et psychologiques et lui donne un caractère vraiment particulier. 


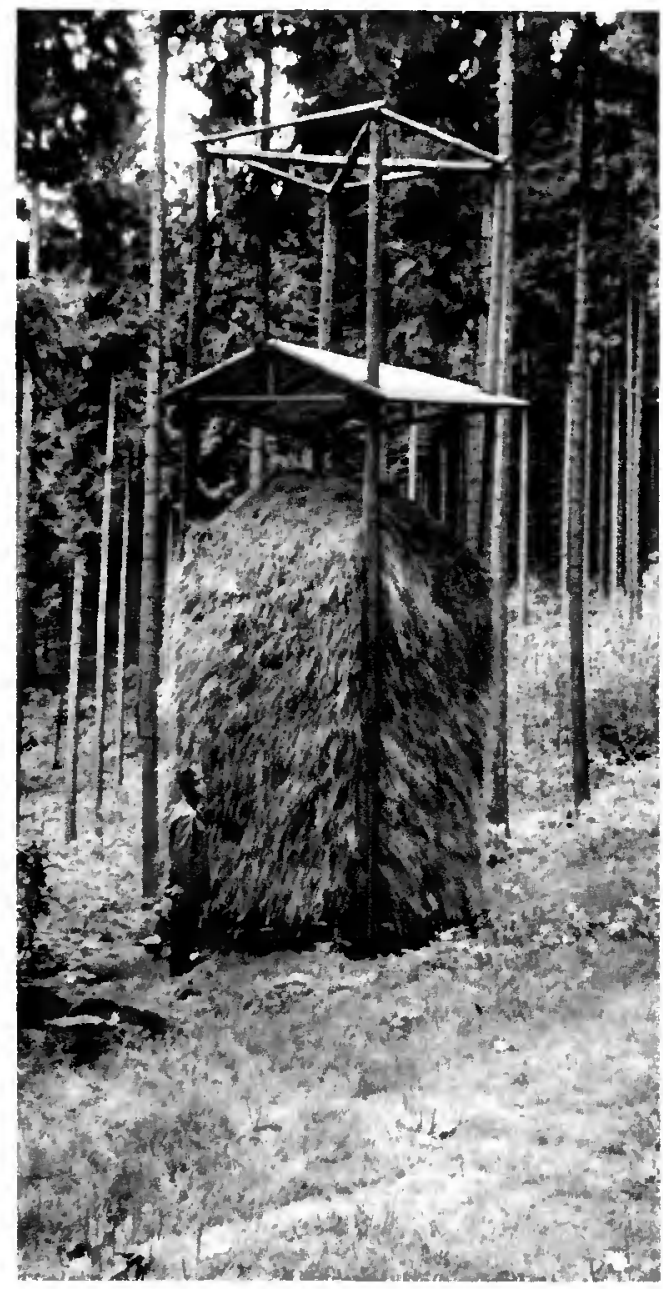

Recherche d'une amélioralion des techniques d'amènagement cynégètique : un dispositif d'affouragement approvisionne en une seule fois au debut de l'hiver supprime les manutentions, et le foin comprime repond mieux qu'en rảtelier aux exigences des animaux. PMOTO DABURON

\begin{abstract}
Ainsi, de nombreux états d' "équilibres sylvocynègétiques " sont-ils concevables pour une même forêt et l'Homme détient le pouvoir d'en fixer le niveau, là où bon lui semble, à la seule condition de satisfaire à un certain nombre d'exigences écologiques et èthologiques des animaux.
\end{abstract}

\title{
Recherche d'un équilibre sylvocynégétlque
}

rout ceci nous montre avec évidence la nécessité, pour une forêt donnée, de définir en premier lieu les critères en fonction desquels l'Homme cherchera à établir et à maintenir tel équilibre plutôt que tel autre.

La recherche de l'équilibre passe donc obiigatoirement par une phase d'orientation, partant de la situation concrète actuelle, au cours de laquelle on fixe lesquels des trois principaux usages possibles de la forêt : production ligneuse, chasse, tourisme, doivent être pris en considération dans le cas particulier envisagè (1). Cette étude préalable doit s'appuyer d'une part sur les potentialités physiques de la forêt dans les trois domaines, d'autre part sur les besoins de l'économie locale ou nationale, les besoins et les pressions des groupements humains locaux, etc...

\section{On passe ensuite à la phase de recherche proprement dite :}

1) Dans la grande majorité des cas, on a affaire à une forêt dont la production ligneuse actuelle ou potentielle est intéressante et oú l'existence d'une ou plusieurs espèces de gibier, techniquement possible, est de plus souhaitable en raison de l'environnement humain.

Deux cas peuvent alors se prësenter : la forêt contient actuellement des quantités appréciables de gibier de certaines espèces ou bien elle est pratiquement vide.

Si elle est vide, on peut songer à des introductions d'espèces adaptées aux conditions de la forêt et, en premier lieu, à celles qui ne causent pas de dommages particuliers aux peuplements, par exemple le faisan en forêt suffisamment ouverte, ou le sanglier. Ces espèces apporteront un revenu supplémentaire à la forêt qu'il faudra comparer aux frais d'entretien, d'affouragement, de gardiennage. On pourra admettre une rentabilité assez faible dans la mesure où la satisfaction des besoins locaux constitue déjà un facteur positif et où la production ligneuse reste la

(1) Nous mettons à part le cas des foréts dont la fonction a un caractère obligatoire, par exemple les foréts de protection, où toute entrave à cette fonction (dégấts aux plantations, piétinement) doit ètre proscrite. 
préoccupation essentielle du gestionnaire. La conception actuelle de la "Vie sauvage " peut cependant pousser à introduire ou réintroduire des Cervidés; des dégâts sont alors à craindre, et l'on passe au cas étudié ci-après.

Si la forêt contient déjà certaines espèces de gibier dommageables aux peuplements (Cervidés), il est peu réaliste, pour des raisons techniques aussi bien que de politique locale, d'imaginer qu'on puisse les supprimer brutalement et les remplacer par d'autres inoffensives.

Compte tenu des considérations imposant dans la forêt un effectif minimum de gibier à chasser, le gestionnaire de la forêt peut adopter plusieurs attitudes :

L'attitude la plus simple, et même simpliste, consiste à fixer un seuil de dégâts admissibles du point de vue sylvicole pour tel ou tel type de plantation ou de régénération naturelle rentrant dans le cadre de l'aménagement (seuil qui devra étre déterminé progressivement par tâtonnements et comparaisons), et de considérer que, si le pourcentage de dégâts constatés dépasse ce seuil, l'effectif des Cervidés est trop èlevé et doit être réduit de façon permanente. On risque alors de ne plus avoir qu'une population de gibier biologiquement inviable et, en outre, de ne plus satisfaire le chasseur qui exige un minimum d'animaux de certaines espèces.

Dans une approche plus fine de la question, étant connu le seuil de dégâts admissibles du point de vue sylvicole, et compte tenu du niveau des dégâts actuels ou à craindre, le raisonne ment conduisant à la fixation de l'état d'équilibre doit faire intervenir, outre une certaine diminution possible du cheptel :

- le coût des mesures de protection éventuelle (protection individuelle par répulsifs, protection globale par engrillagement, etc.);

- le coût des améliorations cynégétiques telles quaffouragements, amélioration de gagnages, pouvant jouer un rôle de "dissuasion " dans la protection des peuplements;

- l'augmentation ou la diminution du revenu à attendre de l'exploitation de la chasse.

Un tel raisonnement purement économique pourrait, à la limite, conduire à accorder théoriquement à la chasse un poids si réduit que, pour des raisons d'ordre biologique ou cynégétique, celle-ci ne soit plus possible. Si, comme on I'a souligné pius haut, les conditions locales et la philosophie de la Protection de la Nature font que la suppression du gibier n'est pas envisageable dans les faits, le gestionnaire n'a alors d'au're solution que d'admettre que la forêt, dans sa totalité, a un rendement moindre que celui qu'il en attendail en pensanî seulement aux arbres qui n'en constituent qu'une partie. II doit, chaque fois que c'est possible, utiliser les techniques de protection individuelle ou de " dissuasion " qui permettent de conserver le maximum d'attrait à la chasse et, par conséquent, d'en tirer le meilleur revenu possible.

A l'inverse, le même raisonnement peut conduire dans d'autres cas à accepter des mesures de protection coûteuses, mais permettant d'admettre un effectif de gibier élevé et, par conséquent, d'en tirer un gros revenu.

2) Un autre cas assez fréquent est celui où les potentialités de la production ligneuse sont faibles ou du moins où l'on n'envisage pour le moment, aucune mesure destinée à accroitre cette production. Les attaques du gibier ne constituent pas alors à proprement parler un dégât, dans la mesure où elles ne compromettent pas l'état boisé que l'on suppose vouloir maintenir : on a seulement affaire à une utilisation plus ou moins intensive des gagnages ou plus généralement du biotope.

Jeune pousse d'Epicea après traitement avec un produit répulsif en automne.

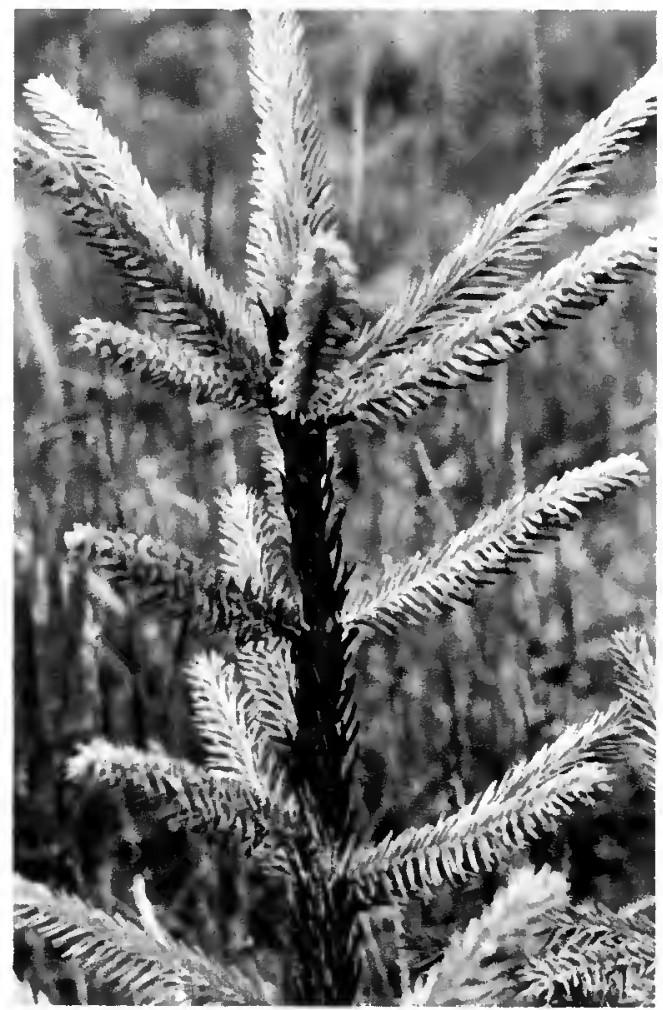


Par exemple un taillis-sous-futaie dégradé, dont l'arnélioration n'est pas possible ou prévue dans l'immédiat, ne sera pas moins rentable si les brins de charme sont abroutis par le cerf que s'ils pouvaient pousser normalement et donner du bois de feu invendable. Si le besoin se tait sentir dans la région de terrains de chasse au chevreuil ou au cerf plus abondants et mieux peuplés, l'objectif unique à adopter est, dès lors, l'objectif chasse qui, seul, pourra rentabiliser la forêt. Ayant choisi les espèces de gibier à favoriser, au vu de leurs possibilités de coexistence, et l'effectif maximum à admettre, compte tenu du biotope et des exigences biologiques de ces espèces, l'équilibre recherchè se fixera en tonction

- du coût des améliorations cynégétiques à apporter (gagnages, affouragements, recépage des taillis, etc.) ;

- du coût des protections minimum éventuellement nécessaires pour maintenir l'état boisé :

- des frais entraînés par l'entretien et l'organisation de la chasse (gardiennage, équipement) et les indemnites éventuelles aux riverains;

- du bénéfice à tirer de l'organisation intensive de la chasse, de la vente des antmaux, etc.

3) A côté des cas très généraux prẻsentés ci-dessus on peut rencontrer dans la recherche de l'équilibre sylvocynégétique des cas particuliers dont l'un, notamment prend une certaine importance : c’est celui de l'équilibre "sylvocynégético-touristique ".

Si la forêt est grande, la zone à usage touristique n'en occupera, en général, qu'une faible partie ou sera étirée le long des axes routiers : un cantonnement géographique des zones à gibier est généralement possible et l'on est ramené aux problèmes précédents. Si la forêt est petite ou fréquentée en permanence sur toute sa surface, le gros gibier se décantonnera et l'exercice de la chasse au petít gibier devra être limité dans le temps. Des considérations d'intèrêt général s'ajoutent ou se substituent alors largement aux aspects de rentabilité développés plus haut.

Nous ne nous dissimulons pas que, dans les lignes qui précèdent, nous avons évoqué des techniques peu familières à beaucoup de gestionnaires de forêts : protection individuelle, affouragements, améliorations de gagnage, ou des notions qui devront être précisées : seuils de dégâts, comptage de dégâts.

Mais qu'on ne s'y trompe pas. Si dans la majorité des forêts de France il n'y a pas d'èquilibre sylvocynégétique, s'il y a trop de dégâts ou pas assez de gibier, si beaucoup de forestiers et de chasseurs poursuivent de sempiternelles querelles, c'est le meilleur indice que les méthodes utilisées jusqu'à présent étaient mauvaises. Il faut donc envisager la question d'un ceil entièrement neuf et avec la volonté d'aboutir. Nous proposons une voie. Peut-être y en a-t-il d'autres meilleures. Puisse au moins celle-ci servir de sentier d'approche!

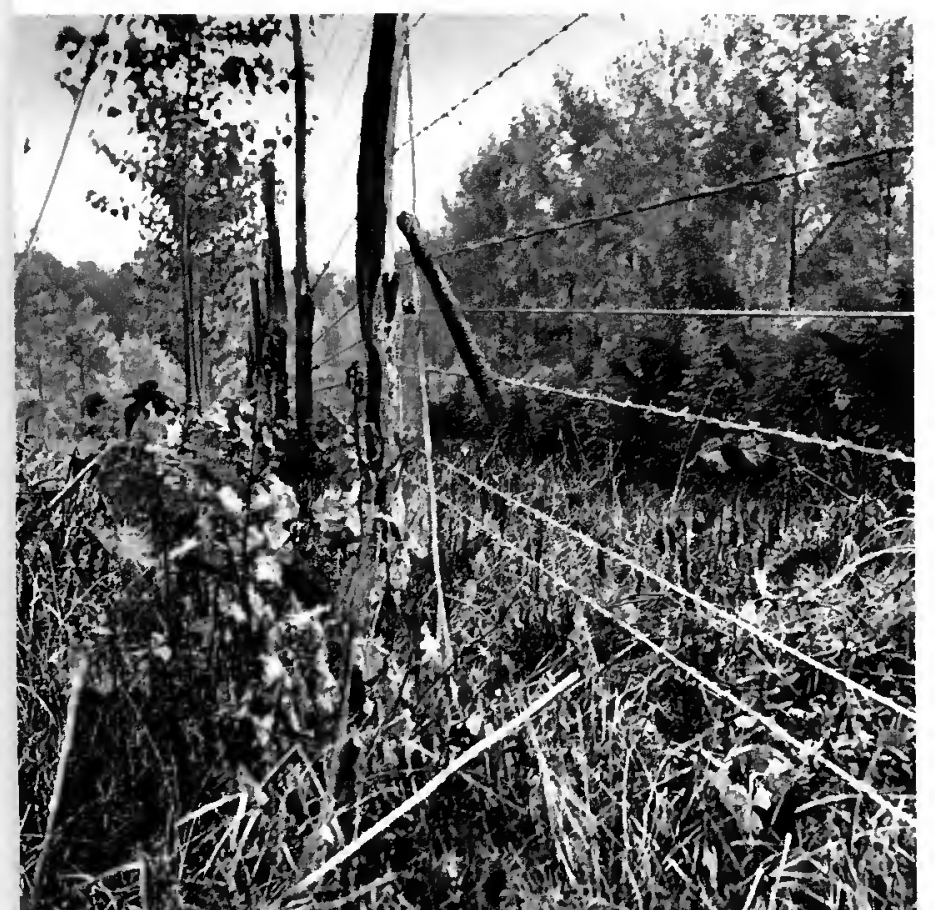

Si elle est systématiquement employée, la clôture est le symbole du déséquilibre cynégétique de la forét.

PHOTO OE L'A 